\title{
Isolation and Identification of Fungi with Glucoamylase Activity from Loog-pang-khao-mak (A Thai Traditional Fermentation Starter)
}

\author{
Nawaphorn Roongrojmongkhon (D), Nattawut Rungjindamai $\mathbb{D}^{\mathrm{D}}$, \\ Tipachai Vatanavicharn (D) and Duangjai Ochaikul* (iD \\ Department of Biology, Faculty of Science, King Mongkut's Institute of Technology Ladkrabang, \\ Bangkok 10520, Thailand.
}

\begin{abstract}
Loog-pang-khao-mak is a Thai traditional fermentation starter that has been used for production of Thai fermented foods for decades. This research aimed to isolate and identify the fungi that produce effective glucoamylase but low ethanol content from the starter. A total of 166 isolates were screened from twelve samples of Loog-pang-khao-mak accumulated from 12 provinces in Thailand using dichloran rose bengal chloramphenicol agar (DRBC Agar). Seventy-nine isolates that effectively hydrolyze starch were selected for glucoamylase activity and alcohol production assay. Three yeast isolates exhibited high glucoamylase activity ranging from 139.14 to 140.94 unit/ml and lowest alcohol yield of $0.41 \%$ (v/v) were Saccharomycopsis fibuligera using morphological and molecular identification. The five isolates of mold exhibited high glucoamylase activity (149.20 to $152.60 \mathrm{unit} / \mathrm{ml}$ ) were identified as Aspergillus niger, Aspergillus oryzae and Amylomyces rouxii. These findings provide further knowledge on the fungi and their potential use as traditional inocula for fermentation of food products.

Keywords: Loog-pang-khao-mak, glucoamylase, alcohol assay, Saccharomycopsis, Aspergillus, Amylomyces
\end{abstract}

*Correspondence: daungjai.oc@kmitl.ac.th

(Received: January 06, 2020; accepted: March 10, 2020)

Citation: Nawaphorn Roongrojmongkhon, Nattawut Rungjindamai, Tipachai Vatanavicharn and Duangjai Ochaikul, Isolation and Identification of Fungi with Glucoamylase Activity from Loog-pang-khao-mak (A Thai Traditional Fermentation Starter), J. Pure Appl. Microbiol., 2020; 14(1):233-246. https://doi.org/10.22207/JPAM.14.1.24

(C) The Author(s) 2020. Open Access. This article is distributed under the terms of the Creative Commons Attribution 4.0 International License which permits unrestricted use, sharing, distribution, and reproduction in any medium, provided you give appropriate credit to the original author(s) and the source, provide a link to the Creative Commons license, and indicate if changes were made. 


\section{INTRODUCTION}

For decades, Loog-pang-khao-mak has been used as a traditional fermentation starter of fermented Thai food and beverage products such as Kao-mak (Sweet fermented glutinous rice), Num Som Saichu (Rice vinegar) and Satoh (Rice wine). This kind of traditional starter has also been used in various other Asian countries with native names such as Nuruk in Korea, Ragi in Indonesia, Murcha in India, Bubod in the Philippines, Fendaqu in China ${ }^{1}$, Ragi-tapai in Malaysia, Koji in Japan and Banh-men in Vietnam ${ }^{2}$. In the preparation of Loog-pang-khao-mak, rice or wheat is mashed and mixed with pepper and garlic. Water is added and thoroughly mixed, and then the solid mixture is shaped into small balls and putted on a weave tray ${ }^{3}$. Traditionally, the powder starter is then dusted over the balls. Then the balls are incubated at room temperature around 2 to 5 days. This kind of fermentation starter is prepared slightly differently in different geographical sources, can be stored for many months ${ }^{4}$. In the fermentation process, Loog-pang-khao-mak works in two steps: saccharification and alcoholic fermentation. It contains a mixture of microorganisms-molds, yeasts and bacteria therefore its fermentative capability is inconsistency. Our main goal was to isolate specific strains of fungi that provide high glucoamylase activity and low alcohol yield which is intended for a low alcohol fermented food product. Rhizopus, Amylomyces, Mucor, Penicillium, Aspergillus, Monascus and Actinomucor are the most common filamentous fungi from Loog-pang-khao-mak ${ }^{2,5}$. A group of yeasts, Saccharomycopsis fibuligera, Wickerhamomyces anomalus, Rhodotorula philyla, Candida tropicalis, Candida rugosa, Issatchenkia orientalis and Saccharomyces cerevisiae are commonly found in this type of starter ${ }^{5,6}$. Molds in Loog-pang-khaomak be able to produce amylolytic enzymes such as glucoamylase and $\alpha$-amylase that can break down starch into glucose and oligosaccharide, while yeasts transform glucose into alcohol. The yeast Saccharomycopsis fibuligera is highly efficient at producing amylolytic enzymes ${ }^{5-8}$.

The purpose of this research was to isolate and identify the molds and yeasts from Loog-pangkhao-mak that provide a high amylolytic activity and a low alcohol yield. The most potential strains from this study can be used as the pure cultures for development of low-alcohol fermentation products as well as static fermentation of Thai fermentation products.

\section{MATERIAL AND METHODS}

Sources of cultures collection of Loog-pang-khaomak samples

All 12 samples of Loog-pang-khao-mak

(a Thai traditional fermentation start) were purchased from local markets in twelve provinces in Thailand (Fig. 1). All samples were crush to flour and stored at $4^{\circ} \mathrm{C}$.

Isolation of molds and yeasts from Loog-pangKhao-mak samples

Ten grams of each sample were resuspended into $90 \mathrm{ml}$ of sterilized water and 10 - fold serial dilutions were performed $\left(10^{-2}\right.$ $\left.10^{-6}\right)$. One hundred microliters from each diluted sample were spread on dichloran rose bengal chloramphenicol agar (DRBC Agar) (Merck). After that the plates were incubated for 7 days at $25^{\circ} \mathrm{C}^{8}$. Colonies with different morphotypes were picked onto potato dextrose agar (PDA) then incubated at the same condition. The fungal culture was maintained in $15 \%$ glycerol at $-80^{\circ} \mathrm{C}$.

\section{Hydrolysis of starch by mold and yeast isolates}

All isolates of yeast and mold were grown on a starch agar and the plates were incubated for 3 days at $30^{\circ} \mathrm{C}$. After that, the iodine solution was flooded onto the plates and the diameters of the clear zone were recorded. The hydrolysis capacity of the isolate was calculated by the following equation reported by Limtong et al. ${ }^{6}$

\section{Alcohol assay of yeast isolates}

Only yeasts with capability to hydrolyze starch were further screened for alcohol production. The selected isolates were grown in yeast peptone dextrose broth (YPD) and the cultured was agitated at $150 \mathrm{rpm}$ and $30^{\circ} \mathrm{C}$ for 18 $h$. Then, cell growth was determined based on the optical density at $660 \mathrm{~nm}$ of the culture broth. Ten milliliters of an inoculum were transferred into $90 \mathrm{ml}$ of YPD broth containing $10 \%$ glucose and incubated under a static condition at $30^{\circ} \mathrm{C}$ for 3 days ${ }^{9}$. Alcohol concentration was analyzed using a gas chromatography (Shimadzu GC-9A, Japan).

\section{Determination of Glucoamylase activity}

Only yeasts and molds exhibiting the capability to hydrolyze starch were screened for glucoamylase activity. Yeasts were grown in 


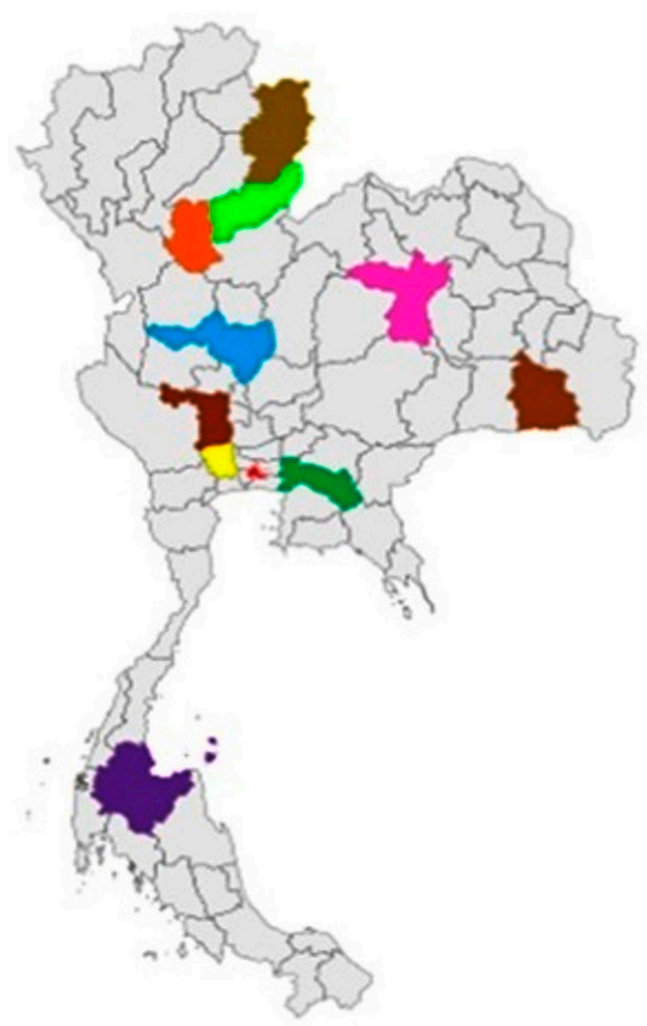

\section{Nakhon Pathom province}

Bangkok province

Suphan Buri province

SuKhothai province

Nakhon Sawan province

Chachoengsao province

Phetchaburi province

$\mathrm{Si} \mathrm{Sa} \mathrm{Ket} \mathrm{province}$

Khon kaen province

Nan province

Uttaradit province

Surat Thani province

Fig. 1. Locations of Look-pang-khao-mak samples were collected in 12 provinces in Thailand.

YPD broth and shaken under the speed of 150 $\mathrm{rpm}$ at $30^{\circ} \mathrm{C}$ for $18 \mathrm{~h}$. The optical density $\left(O \mathrm{D}_{660}\right)$ of the culture was adjusted to 0.5 for inoculum. Ten milliliters of the active cell suspension were transferred to $90 \mathrm{ml}$ of starch broth and shaken under the speed of $150 \mathrm{rpm}$ at $30^{\circ} \mathrm{C}$ for 3 days. Molds were cultivated on PDA for 7 days at $30^{\circ} \mathrm{C}$. A solution of $0.1 \%$ Tween 80 was used to disperse and suspend spores. Ten milliliters of spore suspension $\left(1 \times 10^{6}\right.$ spores $\left./ \mathrm{ml}\right)$ was transferred into $90 \mathrm{ml}$ of starch broth and agitated at $200 \mathrm{rpm}$ and $30^{\circ} \mathrm{C}$ for 3 days. The yeast and mold cultures were centrifuged at 5,000 rpm and $4^{\circ} \mathrm{C}$ for $15 \mathrm{~min}$ and the supernatants were determined glucoamylase activity assay following a method previously reported by Ramadas ${ }^{10}$. One unit of glucoamylase activity was defined as the $\mu \mathrm{mol}$ of reducing sugar (in terms of glucose equivalents) produced per minute under the assay condition

\section{Conventional identification of fungi}

Three most potential isolates of yeasts were identified based on their morphological, physiological, and biochemical characteristics according to the methods reported by Kurtzman and Smith. ${ }^{12}$ as follows. They were cultivated on $5 \%$ malt extract agar (MEA) and incubated for 3 days at $25^{\circ} \mathrm{C}$ and morphological characteristics on MEA were recorded.

For the formation of hypha, the Dalmau plate culture technique was used: each isolate was cultivated on 5\% MEA and incubated for 7 days at $25^{\circ} \mathrm{C}$. The cells were microscopically examined and photographed. Studied on the growth at $37^{\circ} \mathrm{C}$, each yeast isolate was cultivated on yeast malt extract agar (YMA) and then incubated for 3 days at $37^{\circ} \mathrm{C}$.

For an examination of fermentation of carbohydrates, a basal fermentation medium containing $2 \%$ of glucose, sucrose, lactose, galactose, maltose, and trehalose, and $4 \%$ raffinose was used with a Durham tube. The colonies of yeast were suspended in sterilized water, and their turbidity values were estimated by comparing their apparent turbidity to a 


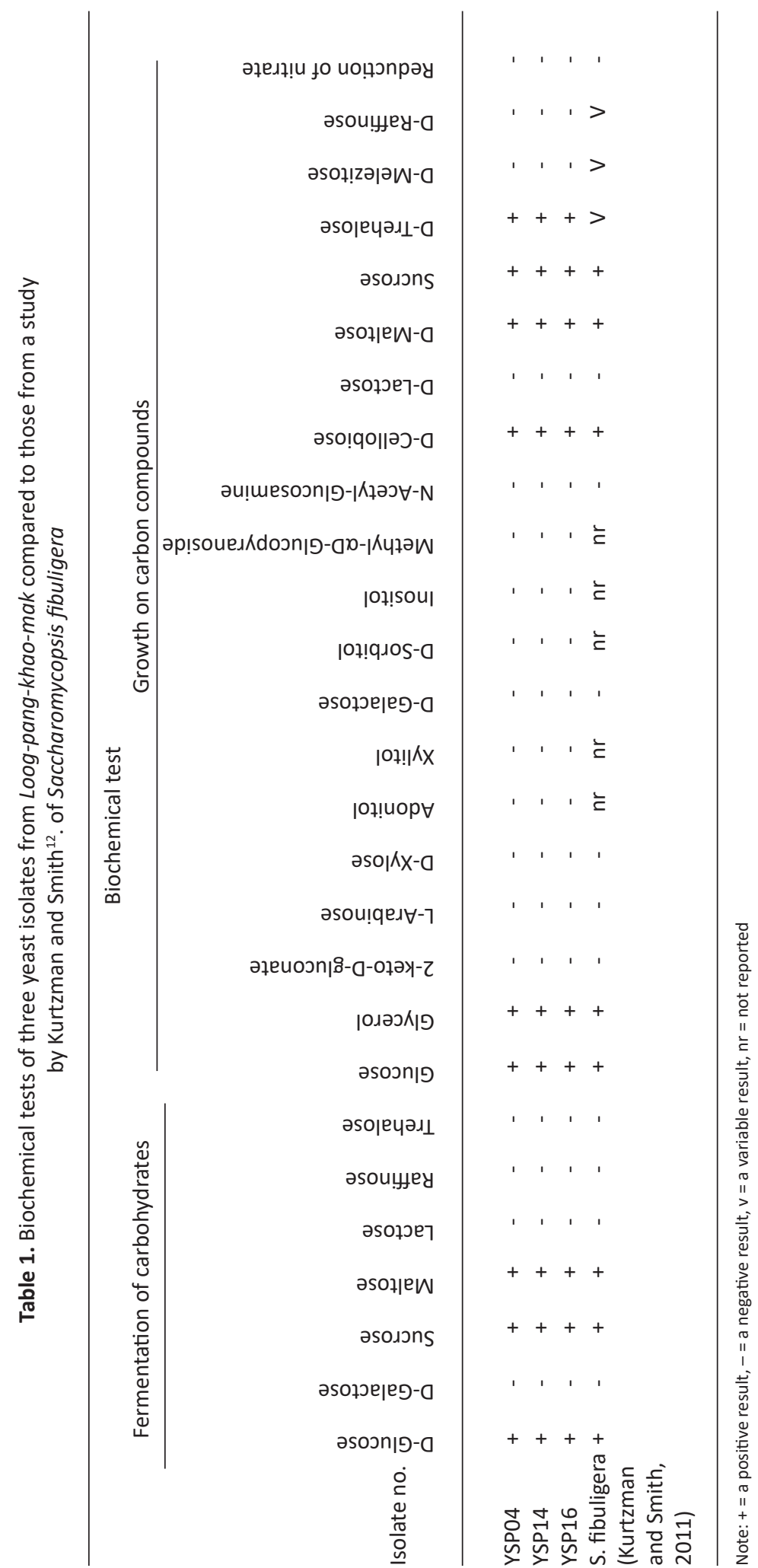


McFarland standard number $3^{13}$. Each test tube was inoculated with the cell suspension then incubated for 7 days at $25^{\circ} \mathrm{C}$. If some gas was detected in the Durham tube in 7 days, the fermentation result was deemed positive. If gas was undetected then, the fermentation result was deemed negative. Assimilation of carbon compounds was determined with an API20C Aux kit (BioMérieux, France).

Five selected isolates of mold were identified based on their morphological characteristics according to the publication by Pitt and Hocking ${ }^{14}$. Mold isolates were grown on three different media: (CYA, MEA and PDA) and maintained for 5 days at $25^{\circ} \mathrm{C}$. The fungal colonies were photographed and their fungal structures were stained with lactophenol cotton blue and microscopically photographed.

The analysis of aflatoxin production on the coconut agar medium (CAM) was studied ${ }^{15}$. Mold isolates were incubated for 5 days at $30^{\circ} \mathrm{C}$. After that the colonies were observed under UV light $(365 \mathrm{~nm})$. Isolates producing aflatoxins on CAM would glow with green fluorescence under UV light.

Molecular identification of the highest potential yeasts and molds

Yeasts were cultured on YPD agar for 1 day at $30^{\circ} \mathrm{C}$. Genomic DNA was extracted using the method followed by Ruiz-Gaitan et al. ${ }^{16}$. Briefly, a few colonies were resuspended into $20 \mu \mathrm{l}$ of 20 $\mathrm{mM} \mathrm{NaOH}$ solution and boiled at $100^{\circ} \mathrm{C}$ for $8 \mathrm{~min}$. For molds, the genomic DNA was extracted using a method previously described by Zhang et al. ${ }^{17}$. The mycelium grown on PDA for 5 days was harvested and resuspended into $100 \mu$ l of sterile water. The content was centrifuged for $1 \mathrm{~min}$ at 10,000 rpm and supernatant was removed. After that, $100 \mu \mathrm{l}$ of a lysis solution $(50 \mathrm{mmol} / \mathrm{L}$ of sodium phosphate at $\mathrm{pH} 7.4,1 \mathrm{mmol} / \mathrm{L}$ of EDTA and $5 \%$ of glycerol) was added and heated at $85^{\circ} \mathrm{C}$. for $30 \mathrm{~min}$. The extracted genomic DNA were stored at $4^{\circ} \mathrm{C}$ until use.

For PCR amplification, $1 \mu \mathrm{l}$ of the genomic DNA was used as a DNA template. A pair of two fungal universal primers for internal transcribed spacer region (ITS) including ITS1 (5'-TCC GTA GGT GAA CCT GCG G-3') and ITS4 (5'TCC TCC GCT TAT TGA TAT GC-3') were used for molecular identification ${ }^{18}$. The PCR cycling conditions were as follows: initial denaturation for $5 \mathrm{~min}$ at $95^{\circ} \mathrm{C}$; followed by 30 cycles of a denaturation for 30 sec at $95^{\circ} \mathrm{C}$; an annealing for $30 \mathrm{sec}$ at $55^{\circ} \mathrm{C}$; an extension for $1 \mathrm{~min}$ at $72^{\circ} \mathrm{C}$ and then a final extension for 5 $\min$ at $72^{\circ} \mathrm{C}$. The amplified products were purified with a FavorPrep ${ }^{\mathrm{TM}}$ GEL/PCR Purification Kit and examined in $1.2 \%$ agarose gel. The purified PCR products were sequenced using the same set of primers. The DNA sequences of the potential fungi were compared with reference sequences available in NCBI GenBank (http://www.ncbi.nlm. hih.gov/blast). A phylogenetic tree based on ITS regions was constructed using MEGA7 Software based on Neighbor Joining (NJ) method.

\section{Statistical analysis}

All data were determined using oneway ANOVA with the SPSS software, version 25 . Significant differences between the means ( $p \leq$ $0.05)$ were analyzed by Duncan's New Multiple Range Test (DMRT).

\section{RESULTS AND DISCUSSION}

Screening of yeasts and molds by starch hydrolysis

All 166 fungal isolates obtained from

12 Loog-pang-khao-mak samples were screened for their capability to hydrolyze starch. Fortytwo isolates of yeasts produced a clear zone with the diameters in the range of $1.99-3.49$ $\mathrm{cm}$. Meanwhile, 37 isolates of molds showed a hydrolysis capacity in the range of $1.00-1.06 \mathrm{~cm}$. According to previously studies indicated that the mold and yeast isolates were found in traditional rice wine starters collected from Vietnam, Nuruk (a traditional starter in Korea), and Starter cakes in India. All these fungi were able to produce the starch degrading enzyme ${ }^{20-22}$.

\section{Glucoamylase activity}

Glucoamylase, which degrades starch and can be found in various microorganisms in particular molds and yeasts, is a very important amylolytic enzyme used in various processes of food industry ${ }^{22,23}$. In this research, 79 fungal isolates were screened for their glucoamylase activity by a dinitrosalicylic acid method, using 1\% starch as the substrate. Three yeast isolates (YSP04, YSP14 and YSP16) showed a high extracellular glucoamylase activity ranging from 139.14 to 140.94 unit/ml (Fig. $2 \mathrm{~A}$ ) with the YSP16 isolate had the highest glucoamylase activity. The 5 isolates of molds (ST02, SR02, UD01, UD02 and PB03) showed a 
glucoamylase activity ranging from 149.20 to 152.60 unit/ml (Fig. 2 B) with the UD01 isolate produced the highest glucoamylase activity at 152.60 unit/ml. Previous studies have reported that amylolytic microorganisms were related to traditional starters used in traditional food fermentation. Some mold and yeast isolates from Loog-pang-khao-mak provided a high amylase activity ${ }^{2,5,6}$. In the same way, Carroll et al. ${ }^{8}$ reported that most mold and yeast isolates found in Nuruk had a strong glucoamylase and $\alpha$-amylase activity similar to Banh men (an alcohol fermentation starter in Vietnam) ${ }^{24}$. Also, mold isolates from Stater cake in India were capable of producing $\alpha$-amylase and glucoamylase ${ }^{22}$.

Alcohol fermentation of yeast isolates

Only yeast isolates were screened for alcohol fermentation. In general, all yeast isolates produced a low alcohol concentration which was less than $1 \% \mathrm{v} / \mathrm{v}$ and three isolates (YSPO4, YSP14 and YSP16) produced the lowest alcohol concentration of $0.41 \% \mathrm{v} / \mathrm{v}$ (Fig. 3). Limtong et al. ${ }^{6}$, which previously reported on yeast isolates from Loog-pang-khao-mak, found that most of the

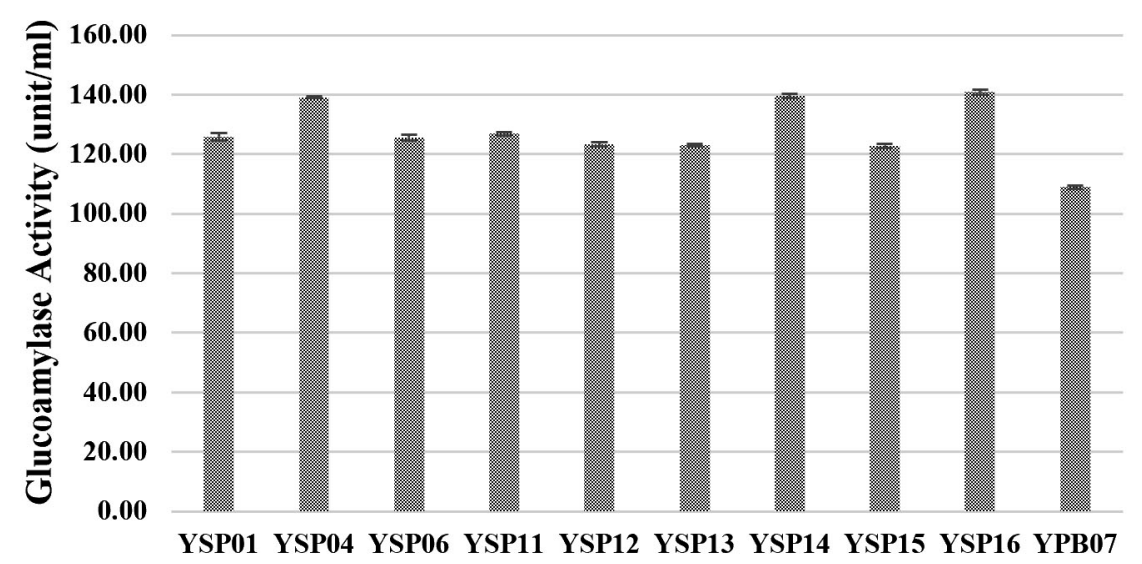

Isolate no.

Fig.2.(A). Glucoamylase activities of fungi were isolated from Loog-pang-khao-mak; (A) Glucoamylase activities of ten yeast isolates

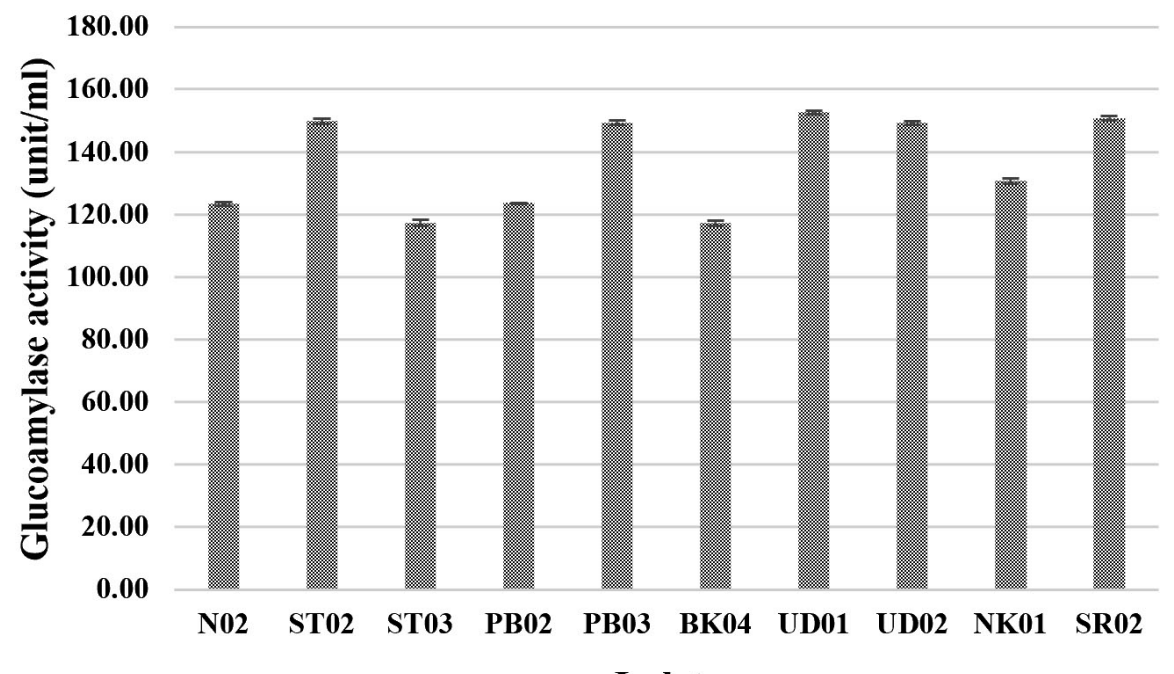

Isolate no.

Fig.2.(B). Glucoamylase activities of fungi were isolated from Loog-pang-khao-mak; (B) Glucoamylase activities of ten mold isolates. 
yeast isolates can ferment $18 \%$ glucose medium into ethanol, and some yeast isolates were able to produce a lower than $2 \%$ ethanol concentration. It is concluded that the yeast isolates yielding a high amylolytic activity could produce low ethanol concentration and vice versa.

Identification of yeasts from Loog-pang-khaomak

Three yeast isolates (YSP04, YSP14 and YSP16) were identified based on morphological and molecular characteristics. On MEA, the three isolates grew into circular, umbonate colonies with off-white to cream-colored mycelia (Fig. 4 A). Budding cells were multilateral, and the formed cells were ovoid to elongate. After 7 days at $25^{\circ} \mathrm{C}$ on MEA, the cells grew into a large number of blastoconidia with true hyphae. All yeast isolates were able to grow at $37^{\circ} \mathrm{C}$. Biochemical tests are shown in Table 1. All three yeast isolates were able to ferment D-glucose, D-maltose and D-maltose. These isolates utilized different carbon sources: glucose, glycerol, D-cellobiose, D-maltose, and sucrose, but none of them was able to assimilate potassium nitrate. Their results were compared to those reported by Kurtzman and Smith ${ }^{12}$, indicating that the three isolates could be Saccharomycopsis fibuligera.

The identity of three yeast isolates was further confirmed by the molecular analysis based on ITS region. They formed a closely relationship with three strains of Saccharomycopsis fibuligera with a high statistical support (100\% NJBS), while other species of Saccharomycopsis were placed in the lower clades (Fig. 4 B), therefore our three isolates (YSP04, YSP14 and YSP16) were identified as $S$. fibuligera. In this study found that $S$. fibuligera strain YSP04, YSP14 and YSP16 exhibited a high glucoamylase activity with $S$. fibuligera strain YSP16 providing the highest activity. S. fibuligera is a dimorphic yeast that is also called Endomycopsis fibuligera ${ }^{12}$. It is widely used in the traditional fermentation starters of various Asian countries such as Loog-pang-khao-mak in Thailand $^{5,6}$, Banh men in Vietnam ${ }^{24}$, Fen daqu in China $^{1}$, Yao qu in China ${ }^{25}$ and Nuruk in Korea ${ }^{8}$. Daroonpunt et al. ${ }^{5}$ reported that $S$. fibuligera isolated from Loog-pang-khao-mak produced the highest glucoamylase activity among various yeast strains similar to Nuruk ${ }^{8}$. Moreover, S. fibuligera strain YSP04, YSP14 and YSP16 were able to produce alcohol less than $1 \% \mathrm{v} / \mathrm{v}$. In contrast, Chi et al. ${ }^{26}$ reported that $S$. fibuligera was unable to ferment ethanol from glucose. Conversely, research by Limtong et $a .^{6}$ presented that $S$. fibuligera produced alcohol content of less than 2 $\% \mathrm{v} / \mathrm{v}$ from $18 \%$ glucose medium at $48 \mathrm{~h}$ and they remarked that it may have other roles in producing a pleasurable flavor.

Identification of molds isolated from Loog-pangkhao-mak

Five isolates (ST02, UD01, UD02, PB03 and SR02) were identified based on their morphological and molecular characteristics. They were cultured on three different media (PDA, MEA and CYA) for

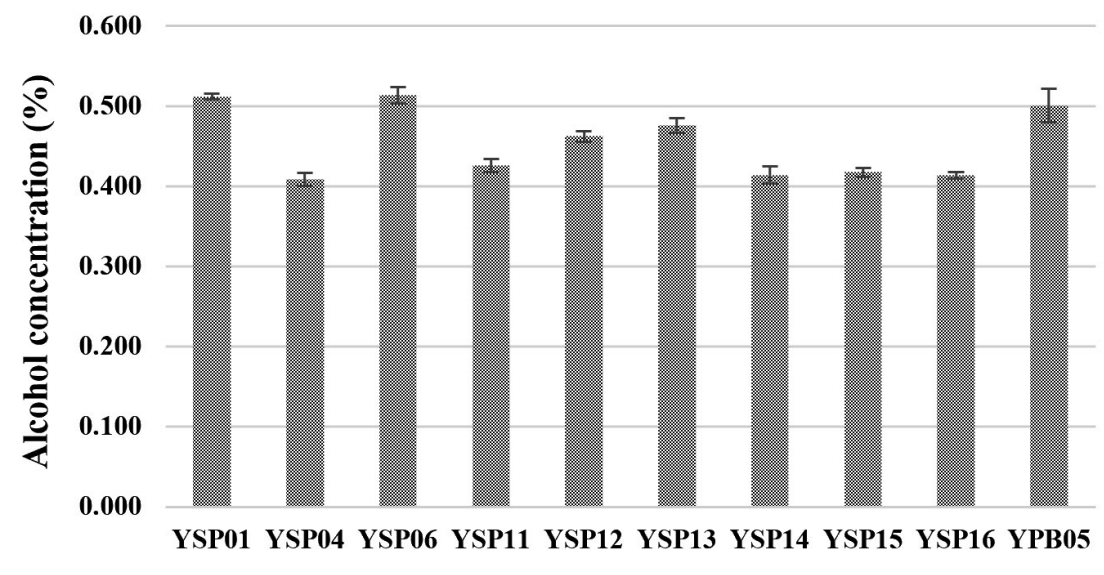

Isolate no.

Fig. 3. Alcohol contents of ten yeast isolates fermented in $10 \%$ glucose medium under a static condition for 3 days incubation. 


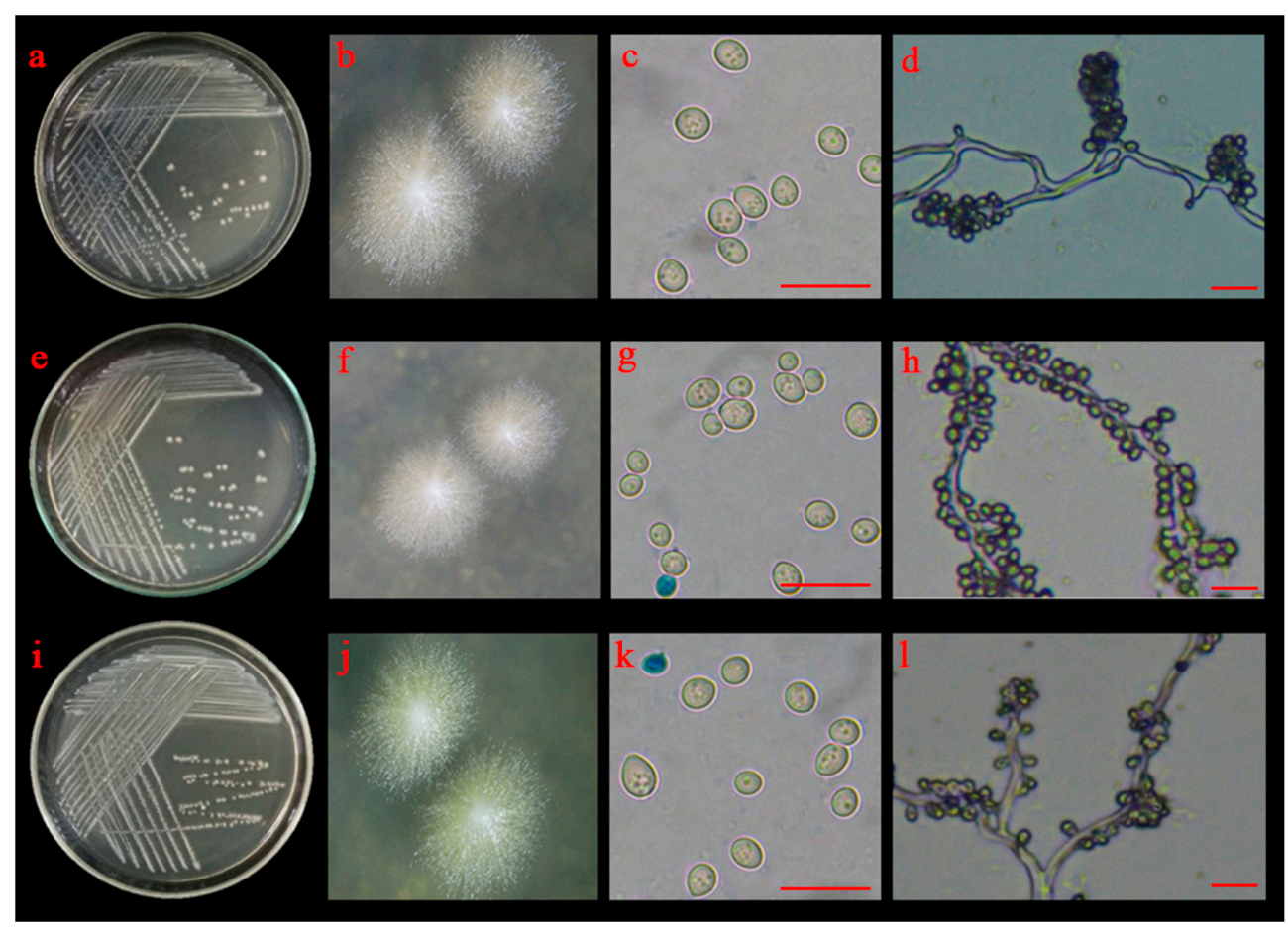

Fig. 4.(A). Morphological characteristics of YSP04; YSP14 and YSP16 isolates: colonies of YSP04 (a and b), YSP14 (e and $f$ ) and YSP16 ( $i$ and j) on 5\% malt extract agar; budding cells of YSP04 (c), YSP14 (g) and YSP16 (k) isolates and blastoconidia with true hyphae of YSP04 (d), YSP14 (h) and YSP16 (I) isolates; the scale bar $=10 \mu \mathrm{m}$.

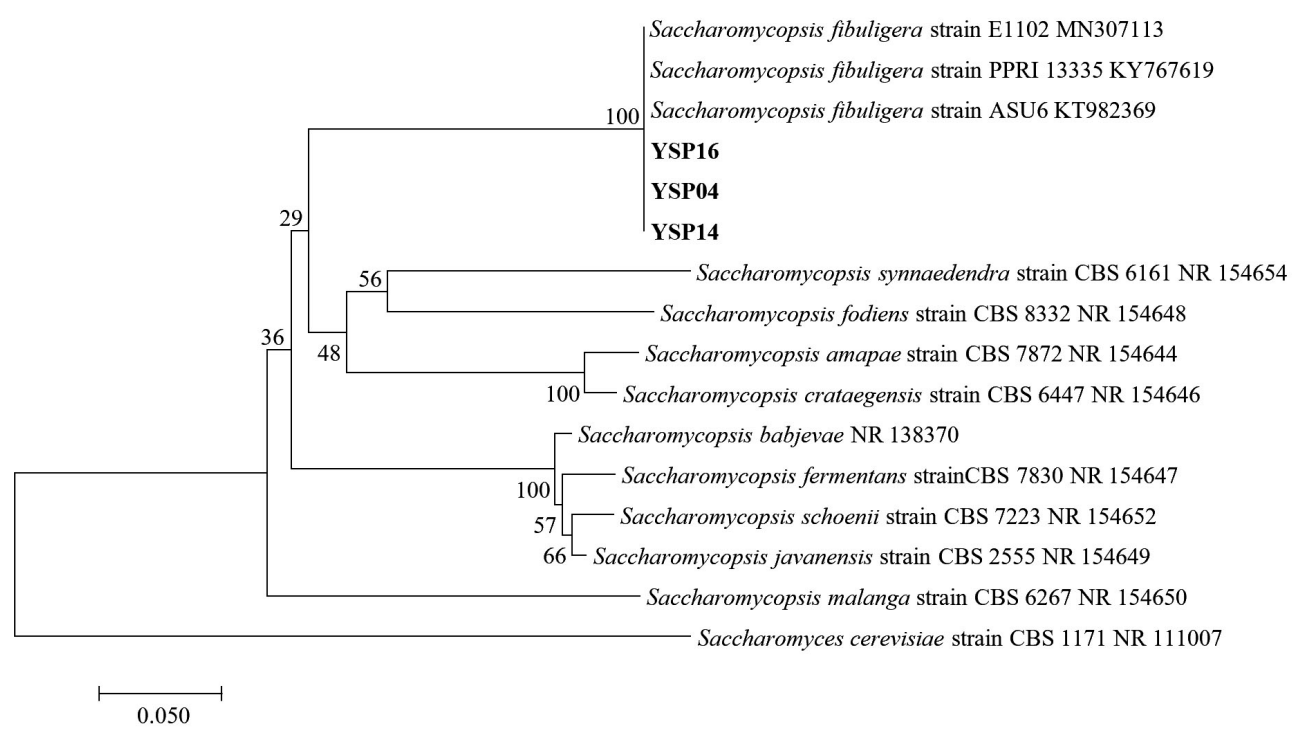

Fig. 4.(B). A phylogenetic tree of 3 isolates of Saccharomycopsis fibuligera (YSP04, YSP14 and YSP16) constructed with the dataset based on ITS gene sequences. The tree was generated from Neighbor Joining Analysis (NJ), and NJ Boostrap (NJBS) values were calculated and shown on the tree. 
5 days at $25^{\circ} \mathrm{C}$. There were three morphotypes of fungal colonies, so their identifications were based on the morphotypes. Although the three isolates (UD02, PB03 and SR02) were cultured on three different media, they produced a similar type of colony (Fig. 5 A, B and C). Colonies were fastgrowing and spreading, dense cottony colonies with white mycelia and chlamydospores were present within 5 days. These chlamydospores were produced in both the substrate and aerial hyphae.

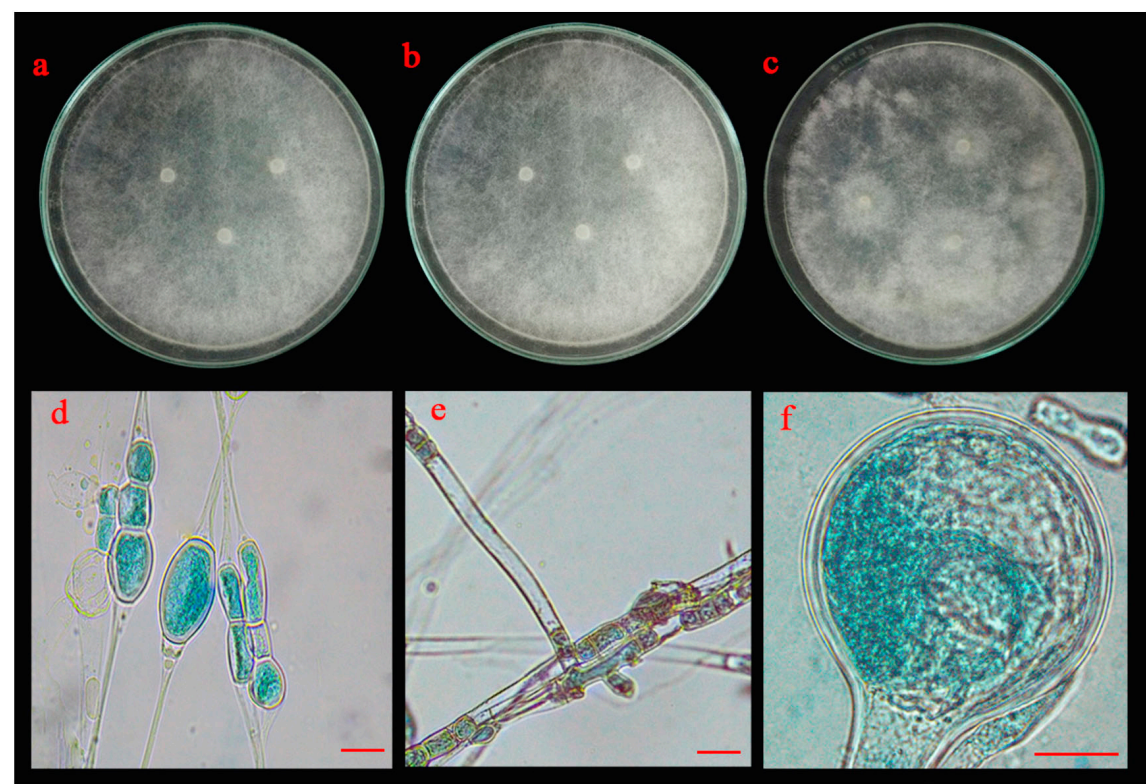

Fig. 5.(A). Morphological characteristics of UD02 isolate: colonies of UD02 isolate on three different media (a; PDA, b; MEA and c; CYA medium); chlamydospores (d), sporangiophore with lake of rhizoid (e) and sporangia and sporangiospore (f); the scale bar $=10 \mu \mathrm{m}$.

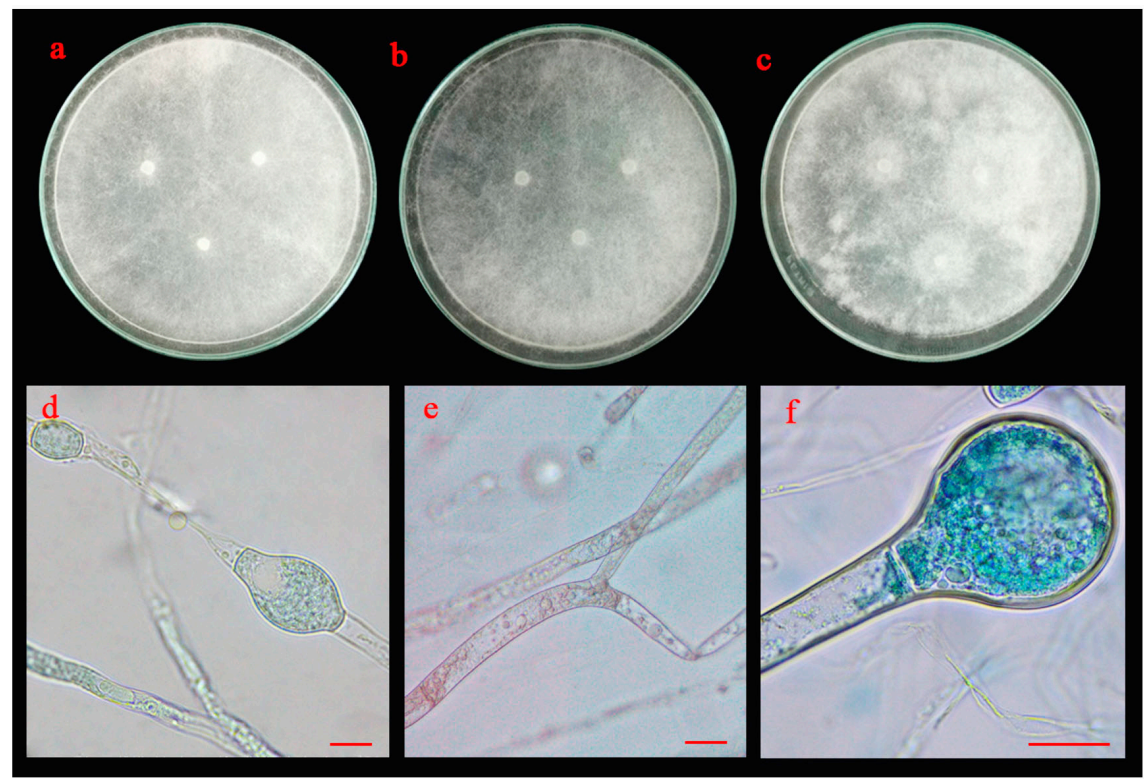

Fig. 5.(B). Morphological characteristics of PB03 isolate: colonies of PB03 isolate on three different media (a; PDA, b; MEA and c; CYA medium); chlamydospores (d), sporangiophore with lake of rhizoid (e) and sporangia and sporangiospore (f); the scale bar $=10 \mu \mathrm{m}$. 
Their spore shapes varied from globose, oval to ellipsoidal. Sporangiophores were also produced in the aerial hyphae. The sporangiophores were hyaline and did not form rhizoids or basal cells (foot cells) with sporangia were globose and the sporangiospores varied from globose to oval.
For the molecular analysis, the three isolates (UD02, PB03 and SR02) formed a clade with three strains of Amylomyces rouxii from GenBank with high support (72 MLBS) (Fig. 5 D) therefore they were identified as Amylomyces rouxii. A. rouxii is a type and only species in the

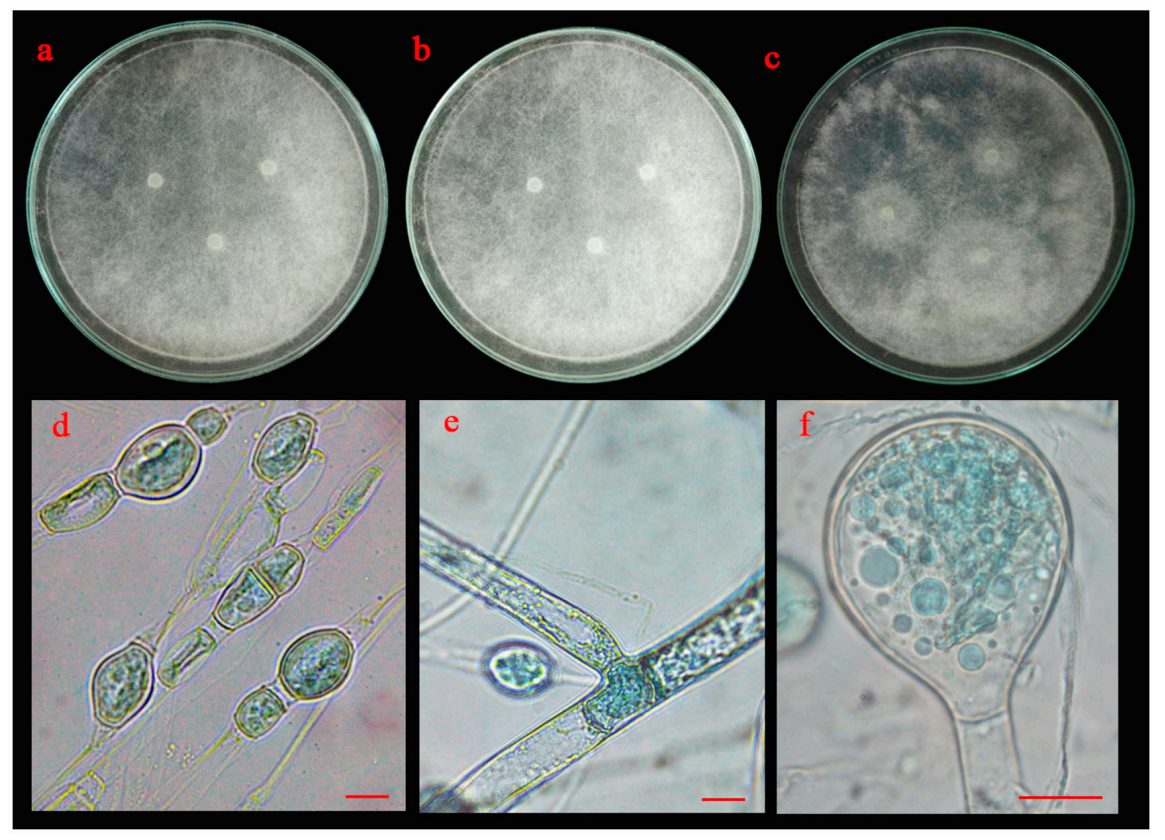

Fig. 5.(C). Morphological characteristics of SR02 isolate: colonies of SR02 isolate on three different media (a; PDA, b; MEA and c; CYA medium); chlamydospores (d), sporangiophore with lake of rhizoid (e) and sporangia and sporangiospore (f); the scale bar $=10 \mu \mathrm{m}$.

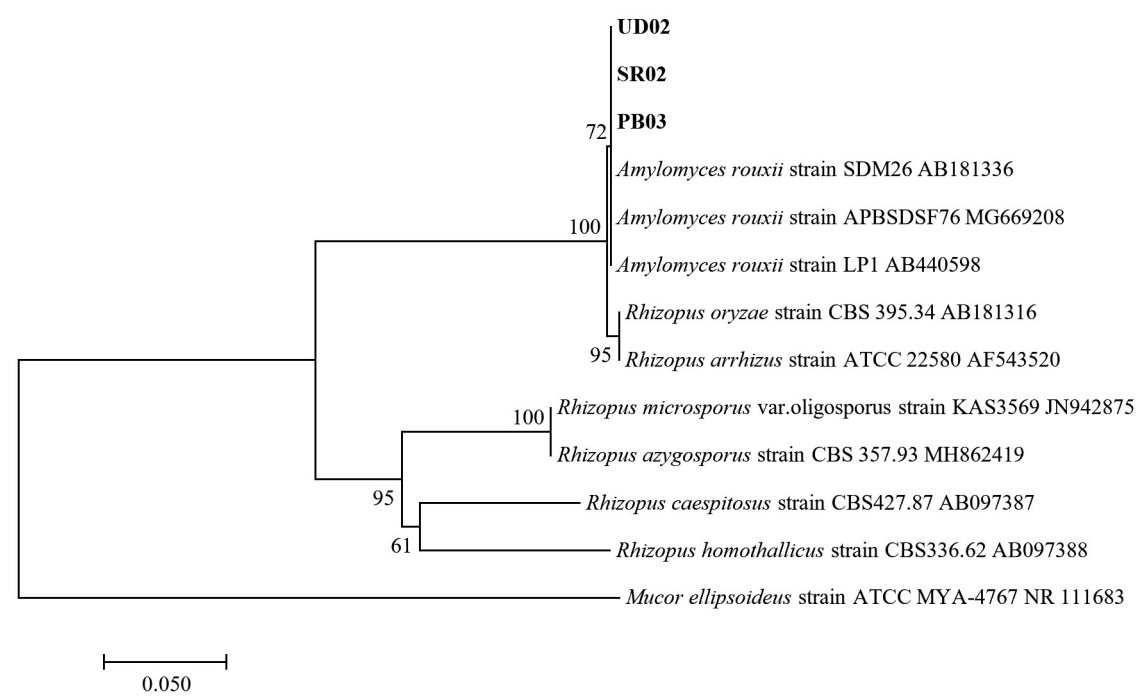

Fig. 5.(D). A phylogenetic tree of 3 isolates of Amylomyces rouxii (UD02, PB03, and SR02) constructed with the dataset based on ITS gene sequences. The tree was generated from Neighbor Joining Analysis (NJ), and NJ Boostrap (NJBS) values were calculated and shown on the tree. 
genus Amylomyces. Although $A$. rouxii is closely related to Rhizopus oryzae ${ }^{27}$, these two genera can be differentiated by their morphological properties $-A$. rouxii produces a large number of chlamydospores but does not form stolon, rhizoids or black sporangia, while Rhizopus oryzae does not produce only a few chlamydospores but forms stolon and rhizoids ${ }^{28}$. The results from our molecular analysis also emphasize the difference between these two genera.

Morphological characteristics of isolate UD01 is shown in Fig. 6 A. On PDA, it produced circular, flat colonies with white mycelia that later developed into greenish-yellow. It grew well on MEA but with different colony type because it produced a flat colony without white margin. It also produced very distinct type of colonies on CYA with velvety colony and olive-green conidia. The conidiophores of the UD01 isolate developed from foot cells and produced globose vesicles with metulae and phialides around the vesicles with yellowish-green conidia on the phialides

Colonies of isolate ST02 are shown in Fig $6 \mathrm{~B}$. Its colonies on PDA were circular, flat colony with white to bright yellow mycelia that later developed into dark brown conidia. On MEA, it grew into a circular, flat colony with white mycelia that developed into black and dusty. Isolate ST02 grew slowly on CYA with cottony and circular colonies, raised colony with white to pale yellow mycelium that developed into pale brown conidia. The conidiophores developed from basal cells (foot cells). It produced globose vesicles with metulae and phialides around the vesicles and pigmented conidia on the phialides.

Isolates UD01 and ST02 had closest relationships with Aspergillus oryzae and Aspergillus niger with statistical supports $67 \%$ and $87 \%$ respectively as shown in Fig. $6 \mathrm{C}$, therefore the two isolates were identified as $A$. oryzae UD01 and $A$. niger ST02. The sequences of rRNA genes of the UD01 strain were closely related to those of $A$. oryzae and $A$. flavus, but they can be differentiated by their aflatoxin production $-A$. flavus produces aflatoxin, while $A$. oryzae does not produce aflatoxin ${ }^{29,30}$. Aflatoxin production of A. oryzae UD01 was undetected when examined with coconut agar medium (CAM) and confirmed its identity as $A$. oryzae.

$A$. rouxii has been used for centuries as a culture starter for production of traditional fermented food and alcoholic beverage in East Asia countries. The use of $A$. rouxii which were reported from Loog-pang-khao-mak in Thailand ${ }^{2,5}$ and Banh men in Vietnam ${ }^{24}$ has been well known to be a producer of amylolytic enzyme ${ }^{31}$. Meanwhile, Aspergillus oryzae and $A$. niger have also been isolated from traditional fermentation starters in

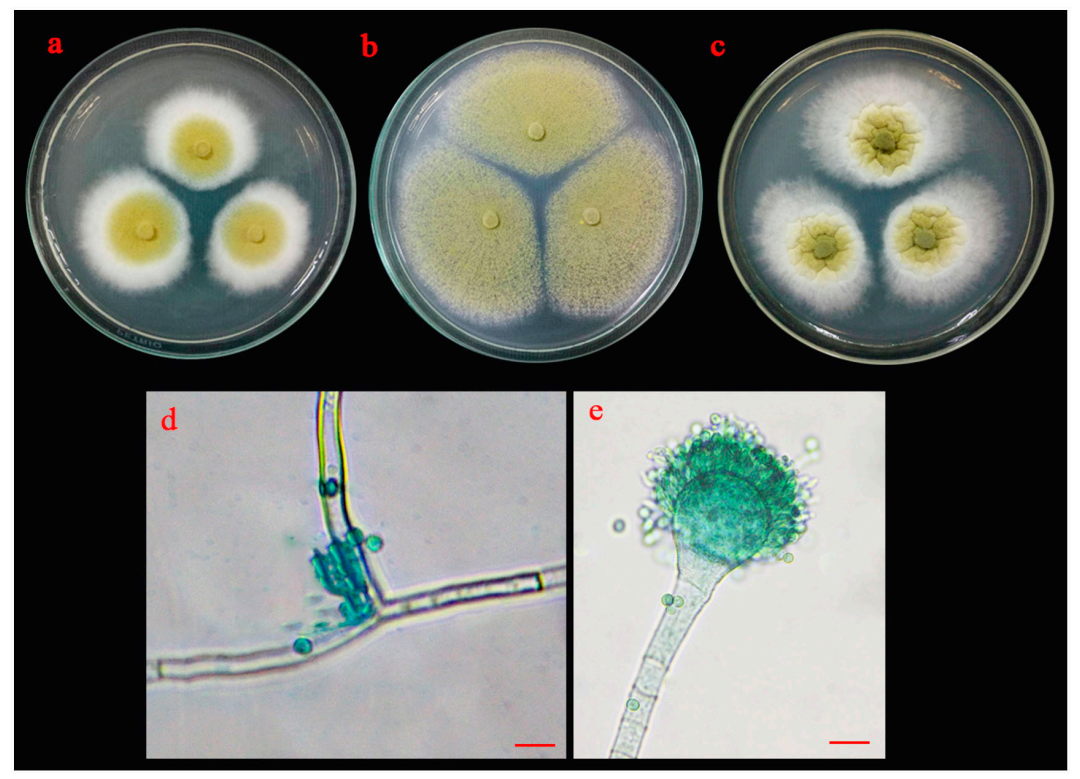

Fig. 6.(A). Morphological characteristics of UD01 isolate: colonies of UD01 isolate on three different media (a; PDA, b; MEA and c; CYA medium); sporangiophore with stolon (d) and conidia (e); the scale bar $=10 \mu \mathrm{m}$. 
various Asian countries such as Loog-pang-khaomak in Thailand ${ }^{2,5}$, Hong qu and Yao qu in China ${ }^{25}$, Koji in Japan ${ }^{32}$ and Nuruk in Korea ${ }^{8,33}$. The two species $A$. niger and $A$. oryzae play an important role for many bio-based industries; for example, they are used for food fermentation, enzyme production, and organic acid production ${ }^{34}$. A. oryzae and $A$. niger are able to produce effective amylase (such as $\alpha$-amylase and glucoamylase) for starch digestion ${ }^{32,35}$. In addition, Jasani et al. ${ }^{36}$ found that $A$. niger have able to produce cellulase enzyme.
Our results indicate that $A$. rouxii PB03, SR02 and UD02, A. oryzae UD01 and $A$. niger ST02 exhibited a high glucoamylase activity. In previous studies of amylolytic fungi associated with starter traditional fermentation, Limtong et al. ${ }^{2}$ and Daroonpunt et al. ${ }^{5}$ presented that $A$. rouxii and Aspergillus spp. were commonly isolated from Loog-pang-khao-mak, and they also noted that $A$. rouxii provided a high amylolytic activity. Carroll et al. ${ }^{8}$ which studied on enzyme activity of fungi obtained from Nuruk found that $A$. oryzae and $A$. niger were able to produce various kinds

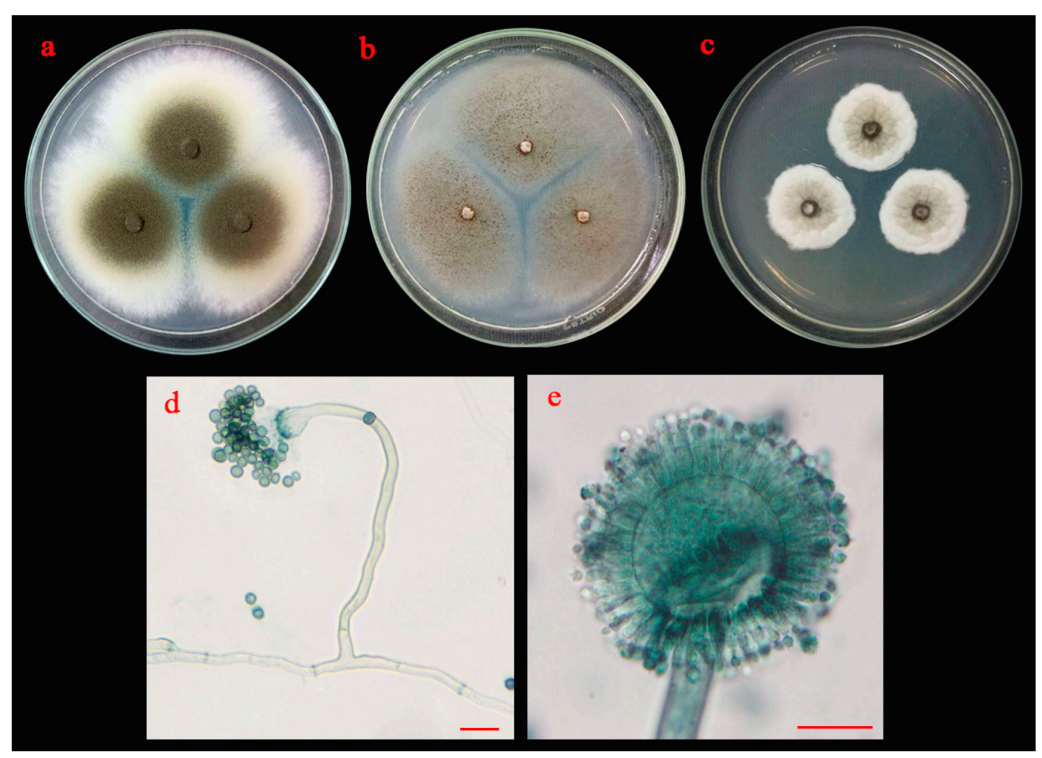

Fig. 6.(B). Morphological characteristics of ST02 isolate: colonies of ST02 isolate on three different media (a; PDA, b; MEA and c; CYA medium); sporangiophore with stolon (d) and conidia (e); the scale bar $=10 \mu \mathrm{m}$.

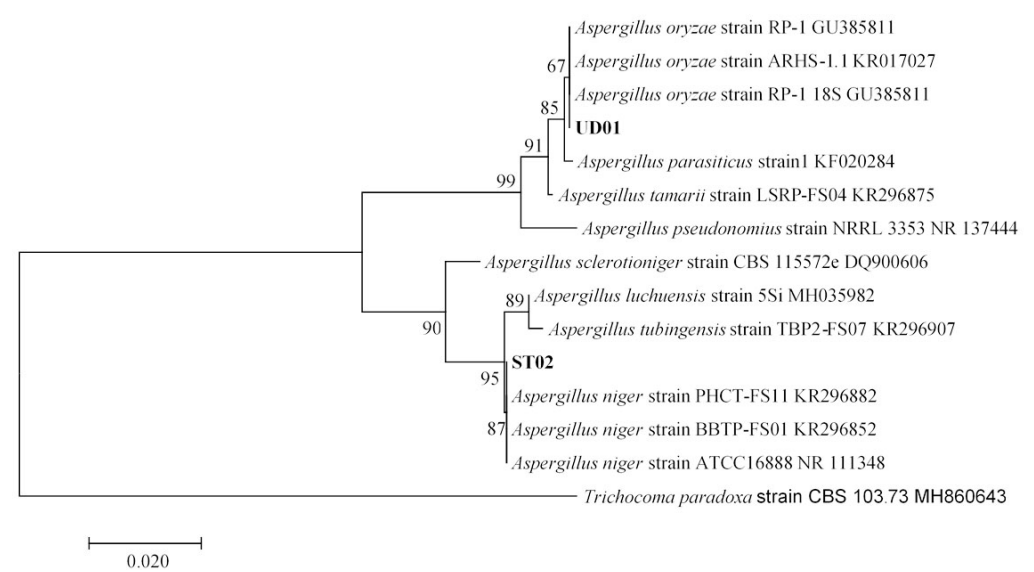

Fig. 6.(C). A phylogenetic tree of Aspergillus oryzae (UD01) and Aspergillus niger (STO2) constructed with the dataset based on ITS gene sequences. The tree was generated from Neighbor Joining Analysis (NJ), and NJ Boostrap (NJBS) values were calculated and shown on the tree. 
of enzymes including glucoamylase, $\alpha$-amylase and acid protease. In particular, $A$. oryzae strain CN1102-08 produced more $\alpha$-amylse and glucoamylase activity than $A$. niger strain LNBSO203. Similarly, the result of this study presents that $A$. oryzae strain UD02 produce glucoamylase activity than $A$. niger strain ST02.

\section{CONCLUSION}

Fungi isolated from Loog-pang-khao-mak (a traditional fermentation starter in Thailand) were screened for their glucoamylase activity and ethanol production. The highest potential isolates were selected and identified based on their morphological and molecular characteristics. Three isolates of yeast, Saccharomycopsis fibuligera YSP04, YSP14 and YSP16, exhibited the highest extracellular glucoamylase activity but with the lowest alcohol yield. For molds, Amylomyces rouxii PB03, UD02 and SR02, Aspergillus oryzae UD01 and Aspergillus niger ST02, gave the highest glucoamylase activity. These findings expand the key knowledge for the inoculum preparation. Using an inoculum from a mixture of pure cultures rather than an inoculum produced by mixture on unknown species which is sold in the market may improve the productivity of traditional fermentation.

\section{ACKNOWLEDGMENTS}

None.

\section{CONFLICT OF INTEREST}

The authors declare that there is no conflict of interest.

\section{FUNDING}

None.

\section{AUTHORS' CONTRIBUTION}

All authors listed have made a substantial, direct and intellectual contribution to the work, and approved it for publication.

\section{DATA AVAILABILITY}

All datasets generated or analyzed during this study are included in the manuscript.

\section{ETHICS STATEMENT}

This article does not contain any studies with human participants or animals performed by any of the authors

\section{REFERENCES}

1. Zheng X-W, Yan Z, Han B-Z, Zwietering MH, Samson RA, Boekhout T \& Robert Nout MJ. Complex microbiota of a Chinese "Fen" liquor fermentation starter (Fen-Daqu), revealed by culture-dependent and culture-independent methods. Food Microbiology., 2012; 31(2): 293-300. https://doi.org/10.1016/j. fm.2012.03.008

2. Limtong S, Sintara S, Suwanarit P, Lotong N. Species diversity of molds in Thai traditional fermentation starters (Loog-Pang). Kasetsart J. (Nat. Sci.)., 2005; 39: 511-518.

3. Wood Brian JB, Lotong N. Microbiology of Fermented Foods, 1998; 658-695. Springer, Boston. https://doi. org/10.1007/978-1-4613-0309-1_21

4. Luangkhlaypho A, Pattaragulwanit K, Leepipatpiboon N \& Yompakdee C. Development of a defined starter culture mixture for the fermentation of sato, a Thai rice-based alcoholic beverage. Science Asia., 2014; 40(2): 125-134. https://doi.org/10.2306/ scienceasia1513-1874.2014.40.125

5. Daroonpunt R, Tanasupawat S \& Keeratipibul S. Characterization and amylolytic activity of yeast and mold strains from Thai sweet rice. Malaysian Journal of Microbiology, 2016; 12(2): 121-131. https://doi. org/10.21161/mjm.73915

6. Limtong $S$, Sintara $S$, Suwanarit $P$ and Lotong $N$. Yeast diversity in Thai traditional fermentation starter (LoogPang). Kasetsart J. (Nat. Sci.)., 2002; 36: 149-158.

7. Saelim K, Dissara Y, Kittikun AH. Saccharification of cassava starch by Saccharomycopsis fibuligera YCY1 isolated from Loog-Pang (rice cake starter). Songklanakarin J. Sci. Technol., 2008; 30(Suppl.1): 65-71.

8. Carroll E, Trinh TN, Son H, Lee Y-W \& Seo J-A. Comprehensive analysis of fungal diversity and enzyme activity in Nuruk, a Korean fermenting starter, for acquiring useful fungi. Journal of Microbiology, 2017; 55(5): 357-365. https://doi.org/10.1007/s12275-0177114-z

9. Lee Y-J, Choi Y-R, Lee S-Y, Park J-T, Shim J-H, Park K-H \& Kim J-W. Screening Wild Yeast Strains for Alcohol Fermentation from Various Fruits. Mycobiology, 2011; 39(1): 33-39.

10. Ramadas M, Holst O \& Mattiasson B. Production of amyloglucosidase by Aspergillus niger under different cultivation regimens. World Journal of Microbiology \& Biotechnology, 1996; 12(3): 267-271. https://doi. org/10.1007/BF00360926

11. Miller GL. Use of Dinitrosalicylic Acid Reagent for Determination of Reducing Sugar. Analytical Chemistry, 1959; 31(3): 426-428. https://doi.org/10.1021/ ac60147a030

12. Kurtzman CP \& Smith MT. The Yeasts, a taxonomic study, 2011; 751-763. $5^{\text {th }}$ Ed(vol.2). Elsevier, London. https:// doi.org/10.1016/B978-0-444-52149-1.00063-X

13. Moore KJ, Johnson MG and Mclary SP. Disk inoculumsolid medium method to test carbon and nitrogen 
assimilation by yeast isolates. J. Appl. Environ. Microbial., 1988; 54: 3185-3186. https://doi. org/10.1128/AEM.54.12.3185-3186.1988

14. Pitt J I \& Hocking AD. Fungi and Food Spoilage. Springer, Boston, 2009. https://doi.org/10.1007/978-0-38792207-2

15. Dyer SK \& McCammon S. Detection of toxigenic isolates of Aspergillus flavus and related species on coconut cream agar. Journal of Applied Bacteriology, 1994; 76(1): 75-78. https://doi.org/10.1111/j.1365-2672.1994. tb04418.x

16. Ruiz-Gaitan AC, Fernandez-Pereira J, Valentin E, Tormo-Mas MA, Eraso E, Peman J \& de Groot PWJ. Molecular identification of Candida auris by PCR amplification of species-specific GPI protein-encoding genes. International Journal of Medical Microbiology, 2018; 308(7): 812-818. https://doi.org/10.1016/j. ijmm.2018.06.014

17. Zhang YJ, Zhang S, Liu XZ, Wen HA \& Wang MA. Simple method of genomic DNA extraction suitable for analysis of bulk fungal strains. Letters in Applied Microbiology, 2010; 51: 114-118. https://doi. org/10.1111/j.1472-765X.2010.02867.x

18. Schoch CL, Seifert KA, Huhndorf S, Robert V, Spouge JL, Levesque CA and Chen W. Nuclear ribosomal internal transcribed spacer (ITS) region as a universal DNA barcode marker for Fungi. Proc. Natl. Acad. Sci (USA), 2012; 109: 6241-6246. https://doi.org/10.1073/ pnas.1117018109

19. Singh S, Singh S, Bali V, Sharma L \& Mangla J. Production of Fungal Amylases Using Cheap, Readily Available Agriresidues, for Potential Application in Textile Industry. BioMed Research International, 2014; 1-9. https://doi.org/10.1155/2014/215748

20. Dung NTP, Rombouts FM \& Nout MJR. Functionality of selected strains of moulds and yeasts from Vietnamese rice wine starters. Food Microbiology, 2006; 23(4): 331-340. https://doi.org/10.1016/j.fm.2005.05.002

21. Kim H-R, Kim J-H, Bai D-H \& Ahn B-H. Identification and Characterization of Useful Fungi with $\alpha$-Amylase Activity from the Korean Traditional Nuruk. Mycobiology, 2011; 39(4): 278-282. https://doi. org/10.5941/MYCO.2011.39.4.278

22. Das AJ, Miyaji T \& Deka SC. Amylolytic fungi in starter cakes for rice beer production. The Journal of General and Applied Microbiology, 2017; 63(4): 236-245. https://doi.org/10.2323/jgam.2016.11.004

23. Slivinski CT, Machado AVL, lulek J, Ayub RA \& Almeida MMde. Biochemical characterisation of a glucoamylase from Aspergillus niger produced by solid-state fermentation. Brazilian Archives of Biology and Technology, 2011; 54(3): 559-568. https://doi. org/10.1590/S1516-89132011000300018

24. Thanh VN, Mai LT \& Tuan DA. Microbial diversity of traditional Vietnamese alcohol fermentation starters (Banh men) as determined by PCR-mediated DGGE. International Journal of Food Microbiology, 2008; 128(2): 268-273. https://doi.org/10.1016/j. ijfoodmicro.2008.08.020
25. Lv X-C, Huang X-L, Zhang W, Rao P-F \& Ni L. Yeast diversity of traditional alcohol fermentation starters for Hong Qu glutinous rice wine brewing, revealed by culture-dependent and culture-independent methods. Food Control, 2013; 34(1): 183-190. https://doi. org/10.1016/j.foodcont.2013.04.020

26. Chi Z, Chi Z, Liu G, Wang F, Ju L \& Zhang T. Saccharomycopsis fibuligera and its applications in biotechnology. Biotechnology Advances, 2009; 27(4): 423-431. https://doi.org/10.1016/j. biotechadv.2009.03.003

27. Kito H, Abe A, Sujaya I-N, Oda Y, Asano K \& Sone T. Molecular Characterization of the Relationships among Amylomyces rouxii, Rhizopus oryzae, and Rhizopus delemar. Bioscience, Biotechnology, and Biochemistry, 2009; 73(4): 861-864. https://doi.org/10.1271/ bbb. 80773

28. Ellis JJ, Rhodes LJ \& Hesseltine CW. The Genus Amylomyces. Mycologia, 1976; 68(1): 131-143. https://doi.org/10.1080/00275514.1976.12019890

29. Chang P-K \& Ehrlich KC. What does genetic diversity of Aspergillus flavus tell us about Aspergillus oryzae?. International Journal of Food Microbiology, 2010; 138(3): 189-199. https://doi.org/10.1016/j. ijfoodmicro.2010.01.033

30. Bhatnagar D, Ehrlich KC, Moore GG \& Payne GA. Encyclopedia of Food Microbiology, 2014; 83-91. 2nd Ed. Academic Press, New York. https://doi. org/10.1016/B978-0-12-384730-0.00012-4

31. Hesseltine CW, Rogers R \& Winarno FG. Microbiological studies on amylolytic oriental fermentation starters. Mycopathologia, 1988; 101(3): 141-155. https://doi. org/10.1007/BF00437031

32. Ichishima E. Development of enzyme technology for Aspergillus oryzae, A. sojae, and A. luchuensis, the national microorganisms of Japan. Bioscience, Biotechnology, and Biochemistry, 2016; 80(9): 16811692. https://doi.org/10.1080/09168451.2016.1177 445

33. Yang S, Choi SJ, Kwak J, Kim K, Seo M, Moon TW \& Lee Y-W. Aspergillus oryzae strains isolated from traditional Korean Nuruk: Fermentation properties and influence on rice wine quality. Food Science and Biotechnology, 2013; 22(2): 425-432. https://doi.org/10.1007/ s10068-013-0097-6

34. Houbraken J, de Vries RP \& Samson RA. Modern Taxonomy of Biotechnologically Important Aspergillus and Penicillium Species. Advances in Applied Microbiology, 2014; 86: 199-249. https://doi. org/10.1016/B978-0-12-800262-9.00004-4

35. Schuster E, Dunn-Coleman N, Frisvad JC \& Van Dijck PW. On the safety of Aspergillus niger -a review. Applied Microbiology and Biotechnology, 2002; 59(45): 426-435. https://doi.org/10.1007/s00253-002$1032-6$

36. Jasani $H$, Umretiya $N$, Dharajiya $D$, Kapuria $M$, Shah $\mathrm{S}$, Patel J. Isolation, Optimization and production of cellulase by Aspergillus niger from agricultural waste. J Pure Appl Microbiol, 2016; 10(2): 1159-1166. 